\title{
INCREASING ENERGY EFFICIENCY OF ARC STEELMAKING FURNACE WITH ELECTROMAGNETIC STIRRING
}

\author{
${ }^{1}$ Sergii TIMOSHENKO, ${ }^{2}$ Aleksii SMIRNOV, ${ }^{1}$ Eduard NIEMTSEV \\ ${ }^{1}$ Donetsk National Technical University, Pokrovsk, Ukraine, stimoshenko155@gmail.com \\ 2 Physics-Technological Institute of Metals and Alloys of National Academy of Science of Ukraine, Kyiv, \\ Ukraine, stalevoz@i.ua
}

https://doi.org/10.37904/metal.2021.4080

\begin{abstract}
Electric arc furnaces (EAF), especially of foundry class, operating with a long-term technological period, are characterized by a high level of energy loss. Intensification of heat and mass transfer in steelmaking bath by pneumatic or electromagnetic (EMS) stirring contributes to increase energy efficiency of the EAF. Influence of bath shape factor (ratio of diameter to depth), which determines heat loss due to melt surface radiation, as well as, EAF energy efficiency, on generated in liquid steel EMS power, hasn't been sufficiently studied. Aim is to improve energy efficiency of the EAF, using EMS, through optimization the bath shape. Technique consists in comparative assessment of specific stirring power by numerical modeling of electro vortex flows in liquid steel at varying bath shape factor. Scientific novelty lays in development the concept of a "deep" bath in relation to EMS. Found that decrease in the bath shape factor from traditional 5 to 2.5 is characterized by growth of power, released during EMS, by 19-36\%, more pronounced with increased current force and reduced current frequency in the stator winding. Geometrical and operating parameters, at which the EMS power exceeds the capabilities of pneumatic stirring, have been determined. Practical value is in 6-9 \% reduction of technological period under EMS conditions in a "deep" bath, which allows decrease of specific power consumption in melting cycle of foundry class EAF by $1.5-2 \%$ and by $5-6 \%$ for the EAF, operating as part of micro-metallurgical complex.
\end{abstract}

Keywords: Arc furnace, electromagnetic stirring, bath geometry, energy efficiency

\section{INTRODUCTION}

Electric arc furnace (EAF) is universal steelmaking unit for both "large" and "small" metallurgy with a possibility of intensive and concentrated energy input, regulation of oxidizing potential in workspace and wide choice of options for initial charge. In foundries of Ukraine there is a certain surplus of the EAF with a capacity of 1.5-6 tons, which arose in connection with current reorientation of sales markets. Some of these units are supposed to be used as a basis of micro-metallurgical plants with a flexible production program [1].

Energy-intensive classical technology significantly limits energy efficiency of foundry-class EAF. The situation is aggravated by traditional ideas about technological expediency of a shallow flat bath with a shape factor (ratio of diameter to depth) of 5.5-4.5, while in converter and ladle-furnace unit it is 1-1.2. Melt surface during technological period is a source of increased radiation heat loss. Therefore, intensification of heat and mass transfer in steelmaking bath by forced mixing is a decisive factor in increasing EAF energy efficiency.

In DC arc furnaces, mixing of the bath is provided by electro vortex flows (EVF) that arise between upper and bottom electrodes in electrically conductive volume of liquid steel [2]. For prevailing in the world industrial frequency EAF the efficiency of EVF is developing mainly within volume, determined by electrodes pitch diameter and depth of electro-dynamic penetration of arcs into the melt, and it doesn't provide effective mixing of the bath. To intensify the processes of heat and mass exchange, forced pneumatic $[3,4]$ or electromagnetic 
stirring (EMS) $[5,6]$ is used. The EMS system was implemented historically earlier, but with the development of porous ceramic plugs technology, pneumatic stirring took priority positions.

Pneumatic mixing power is defined by energy of isothermal expansion of inert gas volume purged into the melt per unit of time [7]. When blowing, two-phase region of reduced density is formed in the bath, which is the driving force of arising circulation. Along with its availability, the method has several disadvantages: local effect and instability of bubble regime in two-phase region in shallow EAF bath. Transition of blowing to jet mode leads to "breakdown" of the bath, in which the efficiency of transfer floating gas volumes potential energy to liquid steel by means of friction forces, as well as the interfacial surface, is sharply reduced.

Main element of the EMS system is a stator located under the bottom, operating at low $(0.2-5 \mathrm{~Hz})$ frequency. Stirring of liquid steel occurs under the influence of the field of volumetric electromagnetic forces, arising from interaction of induced current with its own magnetic field. The advantage of EMS is a non-contact transmitting of stirring power to the melt throughout entire volume of the bath, in contrast to the local nature of pneumatic mixing effect in two-phase region. In the last decade, EMS has received a new development [8]. However, the method is capital and energy intensive.

Reduction of heat loss due to EAF bath surface radiation by $8.5-49 \%$, as studies [8] have shown, can be achieved by decrease of bath shape factor to 2.5-1.8, taking into account structural and technological limitations inherent arc furnaces of various types. At the same time, steel refining rate is expected to be no lower than in a furnace with traditional bath geometry, despite of decrease in metal-slag interface, due to intensification of heat and mass transfer processes under conditions of pneumatic mixing in a "deep" bath.

Influence of the EAF bath shape factor on EMS mixing power has not been studied and is not obvious, as for pneumatic mixing, and therefore, requires additional research.

The aim is to improve energy efficiency of low-tonnage foundry class EAF, equipped with EMS system by means of varying bath shape factor. The method consists in numerical simulation of EVF in steelmaking bath and evaluation of EMS mixing power.

\section{NUMERICAL MODELING, RESULTS AND DISCUSSION}

Efficiency of EMS is characterized by specific mixing power $N_{e m}(\mathrm{~W} / \mathrm{t})$ which is the product of average values of electromagnetic force $F_{e m}(\mathrm{~N})$ and EVF rate $u(\mathrm{~m} / \mathrm{s})$ related per EAF capacity $M(\mathrm{t})$.

$$
N_{e m}=\left(F_{e m} \cdot u\right) / M
$$

Average EVF rate $u(\mathrm{~m} / \mathrm{s})$ in circuit, as a result of the balance of electromagnetic and inertia forces [9], is:

$$
u=j \cdot \delta \cdot \sqrt{\mu_{0} / \rho_{s}}
$$

where:

$j$ - average current density, induced by EMS in the studied circuit of the bath $\left(\mathrm{A} / \mathrm{m}^{2}\right)$

$\rho_{s}$ - liquid steel density $\left(\mathrm{kg} / \mathrm{m}^{3}\right)$

$\delta=c \sqrt{\left[\varepsilon_{0} /\left(\pi \cdot f \cdot \mu_{s} \cdot \sigma_{s}\right)\right]}-$ penetration depth of electromagnetic field into liquid steel $(\mathrm{m})$

$c$ - speed of light $(\mathrm{m} / \mathrm{s})$

$\varepsilon_{0}$ - electrical constant $(\mathrm{F} / \mathrm{m})$

$f$ - working frequency $(\mathrm{Hz})$

$\mu_{s}$ - relative magnetic permeability of liquid steel

$\sigma_{s}$ - electrical conductivity of liquid steel $(\mathrm{S} / \mathrm{m})$ 
To calculate the EMS power, a two-dimensional problem of alternating current electromagnetic field was numerically simulated in ELCUT 6.2 application software package. The problem is solved by finite element method. Electromagnetic phenomena in a liquid bath during the flow of electric current are described by Maxwell's equations, generalized Ohm's law and law of electric charge conservation.

Design features of numerical model of 1.5-t foundry class EAF with calculation blocks (B1-B6) is shown in Figure 1. Physical and geometric characteristics of blocks are given in Table 1.

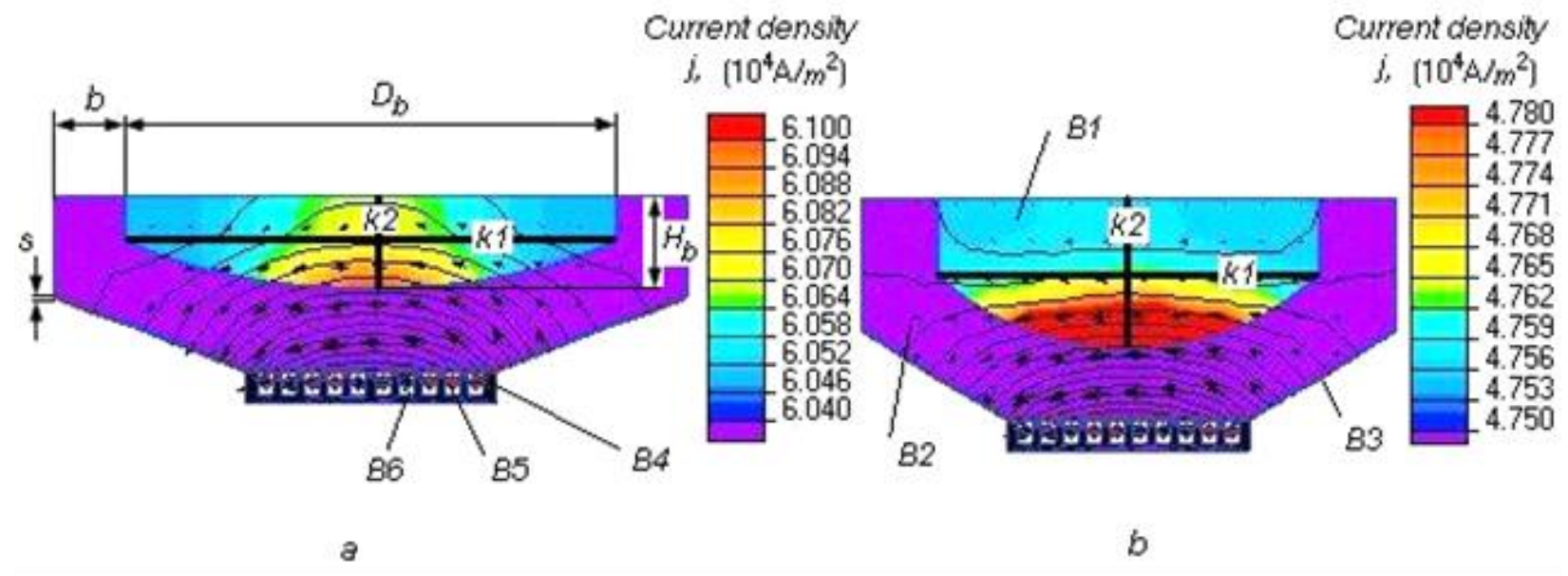

Figure 1 Current density field in base $(a)$ and "deep" $(b)$ bath. Lines and arrows characterize electromagnetic field strength and direction. Designations are in the text

Table 1 Initial data and conditions of numerical modeling of EVF in liquid steel bath

\begin{tabular}{|c|c|c|c|}
\hline \multirow{2}{*}{$\begin{array}{l}\text { Calculation block: material } \\
\text { (designation) }\end{array}$} & \multirow{2}{*}{$\begin{array}{l}\text { Block parameters: electrical } \\
\text { conductivity } \sigma(\mathrm{S} / \mathrm{m}) ; \text { relative } \\
\text { magnetic permeability } \mu \text {; current } \\
\text { force } I(\mathrm{~A}) \text {; frequency } f(\mathrm{~Hz})\end{array}$} & \multicolumn{2}{|c|}{ Dimensions (m) } \\
\hline & & Base bath & "Deep" bath \\
\hline Liquid steel (B1) & $\sigma=7.3 \mathrm{E}+5 ; \mu=1$ & $D_{b}=1.25 ; H_{b}=0.25$ & $D_{b}=1.0 ; H_{b}=0.40$ \\
\hline Refractory lining (B2) & $\sigma=200 ; \mu=1$ & $b=0.20$ & $b=0.20$ \\
\hline Stainless steel casing (B3) & $\sigma=1.1 \mathrm{E}+6 ; \mu=1$ & $s=0.015$ & $s=0.015$ \\
\hline Transformer steel stator (B4) & $\sigma=1.2 \mathrm{E}+6 ; \mu=10^{3}$ & & \\
\hline Copper winding (B5) & $\begin{array}{c}\sigma=5.95 \mathrm{E}+7 ; \mu=1 \\
I=1,500-3,500 ; f=0.25-5.0\end{array}$ & & \\
\hline Air (B6) & $\sigma=0 ; \mu=1$ & & \\
\hline
\end{tabular}

Steelmaking bath is taken to be cylindrical-spherical with the ratio of elements on height equal to 1 . The shape factor $D_{b} / H_{b}$ is 5 for a base bath and 2.5 (taking into account design constraints [8]) for a "deep" one. Current force and frequency in stator winding varied according to Table 1.

Solutions in form of current density fields, carried out at $f=0.5 \mathrm{~Hz}, I=2,500 \mathrm{~A}$ for base and "deep" bath, are shown in Figure 1. Calculating circuits were introduced into obtained solutions: $k 1$ - average horizon of bath, k2 - vertical axis of bath, on which electromagnetic force and current density distribution were determined by means of ELCUT package. Mean values of parameters in given circuits, as the initial data for EMS mixing power evaluation, entered the equations (1), (2).

To compare the capabilities of EMS and pneumatic stirring, ultimate power of the latter $N_{p s}(\mathrm{~W} / \mathrm{t})$ was estimated. It corresponds to the maximum flow rate of inert gas under normal conditions $Q_{g}{ }^{*}\left(\mathrm{~m}^{3} / \mathrm{s}\right)$, at which bubble mode of purging is maintained in EAF bath with hydrostatic height $1.48 \mathrm{~m}$ and temperature $T_{m}(\mathrm{~K})$. 


$$
N_{p s}=\left[\rho_{g} \cdot Q_{g}^{*} \cdot R_{g} \cdot T_{m} \cdot \ln \left(1+H_{b} / 1.48\right)\right] /\left(\mu_{g} \cdot M\right)
$$

where:

$$
\begin{aligned}
& \rho_{g} \text { - inert gas density at normal conditions }\left(\mathrm{kg} / \mathrm{m}^{3}\right) \\
& R_{g} \text { - universal gas constant }(\mathrm{J} /(\mathrm{kmol} \cdot \mathrm{K})) \\
& \mu_{g} \text { - inert gas molecular mass }(\mathrm{kg} / \mathrm{kmol})
\end{aligned}
$$

Regime of purging is defined by geometry of two-phase region in the bath and inert gas flow rate [7, 10]. Criterion of bubble mode for conventionally spherical bubbles is the gas content ratio of two-phase region, not exceeding $\pi / 6$, at which unrestricted motion of bubbles is ensured. The estimation of $Q_{g}{ }^{*}$ for $1.5-\mathrm{t}$ EAF is $2.5 \mathrm{E}$ 04 and $4.4 \mathrm{E}-04 \mathrm{~m}^{3} / \mathrm{s}$ in base and "deep" bath, respectively (for porous plug with diameter of $60 \mathrm{~mm}$ ).

Results of assessment the stirring power in steelmaking bath of 1.5-t EAF with $T_{m}=1,820 \mathrm{~K}$, derived from equations (1-3) using numerical experiment data, are shown in Figure 2. Curves 1, 2 refer to EMS in base and "deep" bath, respectively, at fixed $f=0.5 \mathrm{~Hz}$ (Figure 2a) and fixed $I=2,500 \mathrm{~A}$ (Figure 2b). Lines $1^{*}, 2^{*}$ represent ultimate power of pneumatic mixing in base and "deep" bath, respectively.

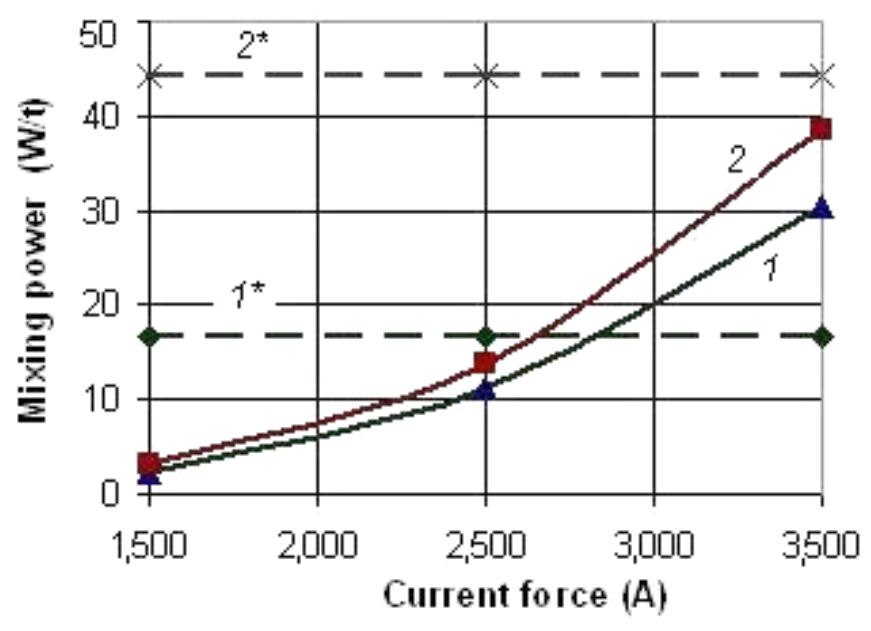

a

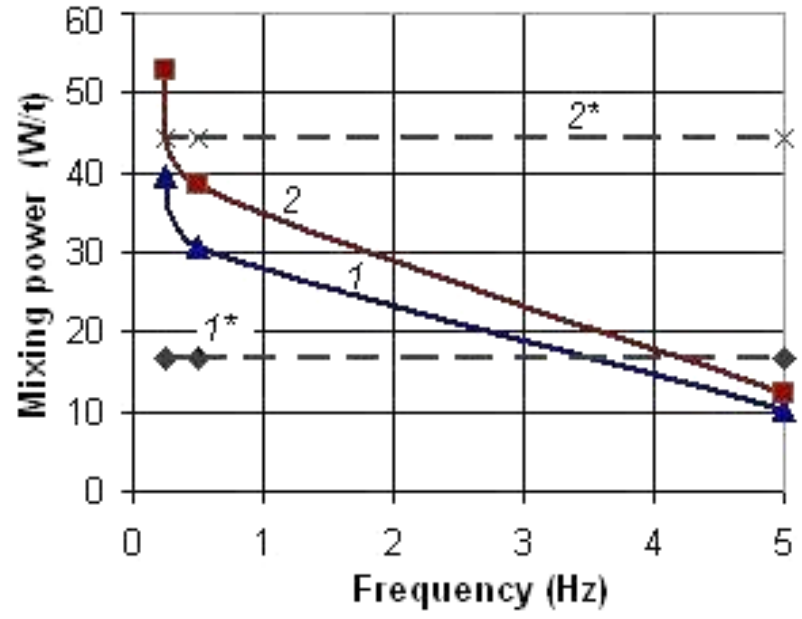

b

Figure 2 EMS power in base (1) and deep (2) bath vs. stator current force (a) and frequency (b). Ultimate pneumatic stirring power in base $\left(1^{*}\right)$ and deep $\left(2^{*}\right)$ bath

According to simulation data, mixing power increases with growth in current force (Figure 2a) and drop in frequency (Figure 2b), which correspond to classical concepts of EMS. Reduction in bath shape factor $D_{b} / H_{b}$ from traditional 5 to 2.5 promotes to increase EMS power by 19-36\%, more noticeable at higher current force and low frequency. Mechanism of phenomenon is, apparently, an increase of magnetic field strength in cross section of "deep" bath with smaller $D_{b}$ at unchanged parameters of stator. This is evidenced by evaluated distribution of electromagnetic energy density over relative bath diameter (circuit k1), shown in Figure 3: in the "deep" bath it increases from $20 \%$ in the central region to $2-4$ times at the periphery in comparison with the base one.

In the base bath at current force more than $2,800 \mathrm{~A}$ and frequency less than $0.5 \mathrm{~Hz}$, power released due to EWF, exceeds ultimate power of pneumatic mixing (for porous plug with diameter of $60 \mathrm{~mm}$ ). In a "deep" bath power capabilities of bottom purging, according to equation (3), are higher, which predetermines parity requirements to EMS, consisting in increased current force or lower frequency: $I=3,500 \mathrm{~A}, f=0.25 \mathrm{~Hz}$. 


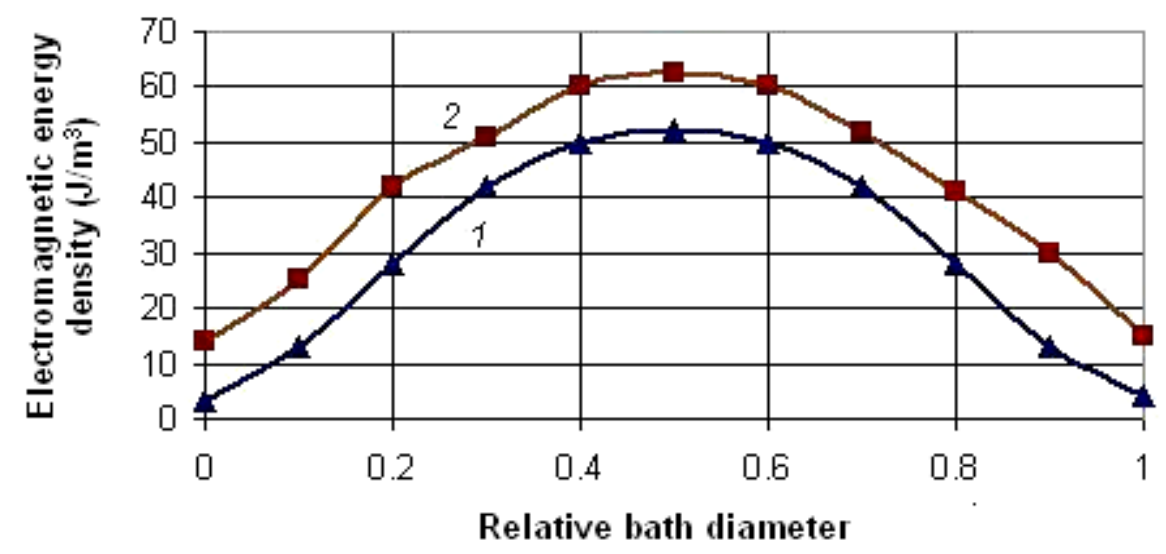

Figure 3 Electromagnetic energy density distribution on middle horizon over relative bath diameter for cases of base (1) and "deep" (2) bath

Duration of the most critical, from the standpoint of EAF energy efficiency, technological period, is determined by interfacial refining, alloying, homogenization and overheating the bath to tapping temperature.

Homogenization time $\tau_{\text {mix }}$ (s) of liquid steel in terms of temperature and chemical composition (with accepted degree of $95 \%)$ is associated with generalized mixing power $N_{\text {mix }}(\mathrm{W} / \mathrm{t})$ by empirical dependence [10]:

$$
\tau_{\text {mix }}=800 \cdot N_{\text {mix }}^{-0.4}
$$

Time of desulfurization of steel $T_{\text {des }}(\mathrm{min})$, from the standpoint of interfacial metal - slag interaction due to mixing power $N_{\text {mix }}(\mathrm{W} / \mathrm{t})$, is determined [11] by initial upon melting [ $\left.S_{0}\right]$ and steel grade [S] content of sulfur (wt. $\%$, and also by chemical complex $\psi=\left(1 / L_{S}\right)\left[M /\left(k_{S l} \cdot M\right)\right]$, including distribution factor of sulfur between metal and slag $L_{s}$ and relative amount of slag $k_{s} /$.

$\tau_{\text {des }}=\frac{\ln \left[\left([S] /\left[S_{0}\right]\right)(1+\psi)-\psi\right]}{-0.031 \cdot N_{\text {mix }}^{0.25}(1+\psi)}$

Homogenization and desulfurization time due to EMS power, according to equations $(4,5)$, in base and "deep" bath of 1.5-t EAF were estimated for the following conditions: $f=0.5 \mathrm{~Hz}, I=2,500 \mathrm{~A},\left[S_{0}\right]=0.046 \mathrm{wt} . \%,[S]=$ 0.025 wt. \%, $L_{s}=50[10], k_{s l}=0.035$. Results are shown in Table 2.

Table 2 Evaluated duration of technological operations in 1.5-t EAF equipped by EMS

\begin{tabular}{|c|c|c|c|}
\hline \multirow{2}{*}{ Technological operation } & \multicolumn{2}{|c|}{ Operation time (min) } & $\begin{array}{c}\text { Relative reduction of } \\
\text { operation time in "deep” } \\
\text { bath (\%) }\end{array}$ \\
\cline { 2 - 4 } & Base bath & 4.7 & 9 \\
\hline Homogenization & 5.1 & 13.4 & 6 \\
\hline Desulfurization & 14.2 & "Deep" bath & 9 \\
\hline
\end{tabular}

Assuming, that desulfurization is limiting stage of technological period in the foundry class EAF, is a reason to expect, focusing on obtained data, a reduction of given period duration by $6-9 \%$ and energy saving regarding the whole heat by 1.5-2\% when using EMS in "deep" steelmaking bath, compared with bath of base geometry.

In the option of EAF application as part of micro-metallurgical complex [1] with intensive melting of semifinished product and relatively short technological period with open bath operation, the loss of radiation heat by melt surface, is expected to be no less than 14-15\% of input energy [12], because of wide application of 
water-cooled elements. This makes it possible to count on decrease of specific energy consumption in conditions of the "deep" bath more than 5-6\%, compared with the base bath.

\section{CONCLUSION}

Numerical study of electric vortex flows under electromagnetic stirring in 1.5-t foundry class EAF shows, that decrease of bath shape factor from traditional 5 to 2.5 promotes growth in electro vortex flows power by 19-36 $\%$, which is more expressed with higher current force and lower frequency of EMS stator.

Reduction of technological period by 6-9\% due to intensification of heat and mass transfer in a "deep" bath with EMS makes it possible to decrease the specific energy consumption in melting cycle of foundry class EAF by $1.5-2 \%$ and by $5-6 \%$ for the EAF, operating as part of micro-metallurgical complex.

\section{REFERENCES}

[1] DUBODELOV, V., SMIRNOV, O., KUBERSKY, S., GORYUK, M. Innovative development of small metallurgical plants as key direction at modernization of steelmaking in Ukraine. Bulletin of Ukraine National Academy of Science. [online]. 2015, no. 12, pp. $33-45$ (in Russian). Available from: https://doi.org/10.15407/visn2015.12.200.

[2] KAZAK, O. Modeling of vortex flows in direct current electric arc furnace with different bottom electrode positions. Metallurgical and Materials Transactions. 2013, vol. 44B, no. 5, pp.1243-1250.

[3] KIRCHEN, M., EHRENGRUBER, R., HANNA, A., ZETTL, K. Latest developments in gas purging systems for EAF. In: AISTech. Proceedings. Cleveland, OH, USA: AISTech., 2015, pp. 1974-1984.

[4] LIU, F., ZHU, R., DONG, K., BAO, X., FAN, S. Simulation and Application of Bottom-Blowing in Electrical Arc Furnace Steelmaking Process. ISIJ International. 2015, vol. 55, no. 11, pp. 2365-2373.

[5] ARZPEYMA, N., WILDLUND, O., ERSSON, M., JONSSON, P. Mathematical Modeling of Scrap Melting in an EAF Using Electromagnetic Stirring. ISIJ International. 2013, vol. 53, no. 1, pp. 48-55.

[6] TENG, L., LJUNGQVIST, P., HACKL, H., ANDERSSON, J. Process improvement with EMS. Steeltimes International. 2016, April, pp. 59-62.

[7] MAZUMDAR, D., GUTHRIE, R. The Physical and Mathematical Modeling of Gas Stirred Ladle Systems. ISIJ International. 1995, vol. 35, no. 1, pp. 1-20.

[8] TIMOSHENKO, S. Computer modeling bath geometry to improve energy efficiency of electric arc furnace. System Technologies. Regional Collection of Scientific Papers. Dnipro: NMetAU, 2016, vol. 3, pp. 33-39.

[9] MIKHAILOV, E., TEPLYAKOV, I. Analytical Solution of the Problem of Electrovortex Flow in Hemisphere with Finite Size Electrodes in Stokes Approximation. Moscow University Physics Bulletin. 2018, vol. 73, no. 2, pp. 162-167.

[10] GHOSH, A. Secondary Steelmaking. Principles and Applications. New York: CRC Press LLC, 2000.

[11] TURKDOGAN, E. Fundamentals of Steelmaking. London: Maney Publishing, 2010.

[12] TOULOUEVSKI, Y., ZINUROV, I. Innovation in Electric Arc Furnaces. Scientific Basis for Selection. Berlin: Springer-Verlag, 2010. 